\title{
Apoyos académicos demandados por estudiantes universitarios. Evidencia para las políticas de apoyos académicos en la educación superior
}

Jorge Valenzuela ${ }^{*}$, Jorge Miranda-Ossandón², Álvaro González-Sanzana ${ }^{3}$ y Carla Muñoz ${ }^{4}$

(1) Facultad de Ciencias de la Educación, Universidad Católica del Maule, Talca, Chile (Correo-e: jvalenzuela@ucm.cl)

(2) Facultad de Educación, Universidad Católica de Temuco, Temuco, Chile. (Correo-e: jmiranda@uct.cl)

(3) Depto. de Educación, Universidad de Magallanes, Punta Arenas, Chile (Correo-e: alvaro.gonzalez@umag.cl)

(4) Departamento de Psicología, Universidad Católica del Maule, Talca, Chile. (Correo-e: cmunozv@ucm.cl)

* Autor a quien debe ser dirigida la correspondencia

Recibido Nov. 28, 2020; Aceptado Ene. 28, 2021; Versión final Feb. 19, 2021, Publicado Jun. 2021

\begin{abstract}
Resumen
Dentro del marco de la creciente demanda por inclusión en la educación superior, el presente artículo tiene por objetivo caracterizar los tipos de apoyos que requieren los estudiantes universitarios a nivel académico para enfrentar con éxito sus estudios. En este estudio, realizado en una muestra de 758 universitarios chilenos, se identifican jerárquicamente los tipos de apoyos que los estudiantes perciben como más necesarios y se analiza la diversidad de apoyos requeridos por estos estudiantes. Los resultados muestran una amplia demanda de todos los tipos de apoyos evaluados, lo que pone en cuestión el enfoque institucional que focaliza los apoyos en aspectos puntuales identificados como carencias e invita a comprender y abordar la problemática de los apoyos académicos de manera más holística y transversal. Se concluye que los apoyos de carácter estratégico instrumental son el principal requerimiento de apoyo en el marco académico por parte de los estudiantes.
\end{abstract}

Palabras clave: ayuda académica; educación superior; servicios universitarios; apoyo académico; estudiantes universitarios

\section{Academic support required by university students: evidence for higher education academic support policies}

\begin{abstract}
This primary aim of this study is to characterize the different types of academic support that university students require to successfully complete their studies. The context is a growing demand for inclusive access to higher education. The sample is composed of 758 Chilean university students. The types of support that students perceive as essential are identified hierarchically and their diversity is examined. The results show high demand for all the academic support types assessed. This puts into question the approach used by educational institutions, which focuses on specific aspects identified as deficiencies, and suggest that academic supportrelated issues should be understood and addressed from a broader, more holistic, and more integrated manner. It is concluded that strategic and instrumental academic supports are the main academic supports required by students.
\end{abstract}

Keywords: academic assistance; higher education; university services; academic support; university students 


\section{INTRODUCCIÓN}

Uno de los fenómenos que ha traído consigo la masificación de las universidades es la llegada de estudiantes que habitualmente no accedían a éstas. En efecto, la composición social, económica y cultural de las universidades chilenas es cada vez más heterogénea, e "ir a la Universidad" para muchos estudiantes supone hoy en día una apuesta por nuevas oportunidades y una promesa de movilidad social. El fenómeno de la masificación de la oferta plantea desafíos que guardan relación con la diversidad de perfiles de los nuevos estudiantes, más que con la cantidad (Hussey y Smith, 2010). Sumado a lo anterior, los estudiantes experimentan un proceso de transición entre adolescencia tardía y adultez emergente en donde se debaten entre la necesidad de autonomía y la necesidad de apoyos tangibles para iniciar la etapa de ser adultos (Barrera y Vinet, 2017), en un proceso subjetivo de cambio y reacomodo que muchas veces se asemeja a un verdadero "salto al vacío" (Rascovan, 2016), del cual las instituciones universitarias deben hacerse cargo (Engstrom y Tinto, 2008).

Ante este escenario, las nuevas generaciones de estudiantes que ingresan a la Universidad, se encuentran frente a una serie de nuevas demandas académicas y que, en muchos casos, no pueden asumir solos (Chang et al., 2020). En particular, en el caso de las dificultades académicas, éstas se pueden afrontar de dos maneras: o se abordan en solitario o se busca ayuda o apoyo. En esta segunda línea, las iniciativas de apoyo académico por parte de las instituciones de educación superior (talleres, cursos, tutorías) han sido un avance (Parnes et al., 2020). Sin embargo, pareciera que no son suficientes para responder a la complejidad de la demanda, cuya magnitud desconocemos (cf. Jeffery y Johnson, 2019).

Por apoyos académicos institucionales nos referimos a un conjunto de iniciativas que las universidades desarrollan para proveer ayuda complementaria a los estudiantes a fin de que ellos puedan avanzar en sus estudios universitarios. Estos, según la institución, poseen distintos formatos, extensión y voluntariedad e incluyen cursos, talleres y tutorías, entre otros, tanto en modalidad individual como grupal. En algunos casos están dirigidos por profesionales, y en otros, por pares calificados. Pese a que en ciertos casos estos apoyos son obligatorios para los estudiantes de primer año, la gran mayoría de estos son voluntarios e implican la búsqueda de ayuda por parte del estudiante.

La literatura sobre búsqueda de ayuda (Help Seeking) es bastante profusa, dando cuenta de una serie de aspectos y factores asociados (Chang et al., 2020; Chowdhury y Halder, 2019). Cabe destacar, en este sentido, que si bien la búsqueda de ayuda es una estrategia de aprendizaje individual, esta búsqueda involucra a otras personas, por lo cual es además, eminentemente, un proceso de interacción social (Black y Allen, 2019). La investigación ha identificado la búsqueda de ayuda académica como un proceso multidimensional que resulta de gran importancia para promover el aprendizaje de los estudiantes y resolver dificultades con el apoyo de otros (Puustinen, 2013). Más allá de la distinción entre tipos de búsqueda de ayuda instrumental y ejecutiva (Chowdhury y Halder, 2019), lo cierto es que hoy el proceso de búsqueda de ayuda es considerado como una estrategia de aprendizaje autorregulada, en la que se movilizan distintos tipos de competencias y recursos por parte del individuo.

Dentro de las variables relacionadas con la búsqueda de ayuda, destacan tanto factores personales como contextuales. En relación a los primeros, cabe destacar los factores motivacionales y emocionales, tales como, por ejemplo, la autoeficacia social y académica, la integración social, la integración académica, o la vergüenza. En relación a los factores contextuales, la identificación con los pares, el tipo de prácticas docentes y actividades impulsadas en el aula, así como las características de los apoyos académicos institucionales, se constituyen en elementos claves para explicar el proceso de búsqueda de ayuda por parte de los estudiantes.

En el contexto universitario, las ayudas provienen no sólo de los pares o agentes más cercanos, sino que también de las instituciones, quienes, conscientes de esta nueva configuración del estudiantado, han generado dispositivos de apoyo a nivel institucional (Parnes et al., 2020). Evidentemente, una primera condición para plantearse la posibilidad de solicitar o aceptar este tipo de ayuda institucional son las creencias asociadas respecto de la utilidad de buscar apoyo (Seyi-Oderinde, 2020). Ello es relevante puesto que la sola necesidad de apoyo pareciera no ser suficiente. La investigación señala que los estudiantes que más necesitan ayuda a menudo evitan buscarla con el objeto de no levantar sospechas respecto de las capacidades personales para asumir o responder a los desafíos académicos o sociales que implica la vida universitaria (Peeters et al., 2020; Seamark y Gabriel, 2018).

En el proceso de búsqueda de ayuda en contextos académicos e institucionales, confluyen dos dimensiones que deben coordinarse para que esta búsqueda de ayuda se concrete. La primera, asociada a la disposición del propio sujeto a buscar ayuda y la segunda respecto de las respuestas que las instituciones generan frente a esta necesidad de ayuda. La confluencia de estas dos dinámicas es condición necesaria para un apoyo efectivo por parte de las instituciones. 
Respecto de la disposición de los sujetos a buscar ayuda, destacan los factores de tipo contextual, entre los cuales, el conocimiento de la existencia de apoyos institucionales es una primera condicionante. Otra, es el conocimiento específico de los procedimientos para solicitar estos apoyos. Evidentemente, no basta con conocer la existencia de los apoyos institucionales, también es necesario identificarse como potencial destinatario, a la vez que valorarlos en términos de importancia y utilidad y saber cómo acceder a ellos.

Por su parte, una condición para que puedan concretarse los apoyos institucionales de manera efectiva, es la "sintonía" entre las necesidades percibidas por los estudiantes y la oferta institucional. Para que el apoyo sea efectivo, las carencias o necesidades objetivas tienen necesariamente que dialogar con la percepción del estudiante sobre aquellos aspectos que él o ella considera que deben ser apoyados. En otras palabras, no basta con identificar los contenidos, habilidades, estrategias que los estudiantes deben adquirir o fortalecer, sino que se requiere conocer, desde la perspectiva del estudiante, qué tipos de ayudas académicas son percibidas como necesarias y valiosas. Esta disposición motivacional es clave para una búsqueda autorregulada de ayuda (Chowdhury y Halder, 2019).

Lo anterior implica una serie de desafíos considerables para los sistemas de apoyo académico institucionales. En primer lugar, lograr que los estudiantes que más lo necesitan, recurran a éstos. En segundo lugar, ser capaces de reconocer las variables motivacionales y emocionales que favorecen o impiden que los estudiantes se acerquen a ellos y que influyen en la disposición de éstos a utilizar la ayuda disponible. Finalmente, en un contexto más amplio, anticiparse a las necesidades de los estudiantes y promover de manera decidida el aprendizaje colaborativo y autorregulado (Collins y Sims, 2006). El presente estudio, en este marco, tiene por objetivo caracterizar los apoyos académicos requeridos por estudiantes universitarios para enfrentar con éxito sus procesos de formación. Se busca establecer una jerarquía de los tipos de apoyos requeridos y evaluar la amplitud de dichas necesidades.

\section{OTROS ANTECEDENTES}

El ingreso a la universidad implica la entrada a una nueva cultura moldeada disciplinariamente que implica, para el estudiante, un proceso de transición hacia la vida adulta, y esto independientemente de su puntaje en las pruebas de selectividad universitaria. El individuo, en este contexto, se transforma progresivamente en estudiante universitario, en un proceso de aprendizaje permanente e integración académica y social conocido como afiliación (Morandi et al., 2019), indispensable para perseverar y tener éxito en los estudios universitarios (Chrysikos et al., 2017). En nuestro país, sabemos que este proceso de transición puede ser dificultoso para muchos estudiantes, particularmente aquellos provenientes de contextos socioeconómicos más desfavorecidos (Silva, 2020). En este nuevo escenario, que constituye la universidad, se hace necesario contar con nuevas habilidades y recursos para adaptarse a los procesos de transición a la vida universitaria (Parnes et al., 2020).

Las necesidades planteadas por estudiantes de primer año dan cuenta de que los tipos de apoyos requeridos, son diversos y multidimensionales, y no se limitan a un tipo específico. En efecto, estos apoyos abordan aspectos de orden personal y contextual (Casanova et al., 2018), pudiendo ser de tipo estratégicos (gestión de tiempo y del estrés, técnicas y hábitos de estudios), disciplinares (conocimientos generales y específicos) y de carácter socioemocional (apoyos afectivos y de salud mental). Cabe destacar que estas necesidades de apoyo pueden permanecer a lo largo de la formación.

\section{Apoyos estratégicos}

La universidad, hoy más que nunca, es el espacio de lo diverso donde se espera que el conocimiento sea puesto en cuestión y revisado de manera permanente desde los avances de la disciplina o desde perspectivas teóricas en competencia. Por lo anterior, ingresar a este nuevo mundo implica, para el estudiante, descubrir una nueva manera de apropiarse y generar conocimiento. A ello, se suma el proceso interno de transición que experimenta el estudiante en su tránsito de la enseñanza secundaria a la universidad (Coertjens et al., 2017). Durante este proceso, las prácticas y dinámicas propias del trabajo escolar pueden no necesariamente resultar eficaces, y aspectos tales como los hábitos y técnicas de estudio, o la gestión del tiempo y del estrés (Halgravez et al., 2017) se vuelven aspectos gravitantes para el éxito académico (Bickerdike et al., 2016; Schneider y Preckel, 2017). En este nuevo contexto, ya no basta con que la universidad establezca ciertos niveles de exigencia académica a los estudiantes, sino que exige de parte de la institución hacerse cargo formativamente de los contenidos y habilidades necesarias para la adquisición de la formación a nivel universitario, tendiendo puentes entre estas necesidades y los apoyos pertinentes para la consecución del plan de formación escogido por el estudiante (ver, por ejemplo, los talleres de apoyo focalizados en habilidades académicas como la gestión de tiempo y del estrés, en http://rendimientoacademico.uc.cl/). 


\section{Apoyos disciplinares}

En este nuevo contexto que constituye la educación superior, no necesariamente el alumno dispone de todos los conocimientos requeridos previamente para andamiar los saberes de la nueva disciplina (Etxeberria et al., 2017; Micin et al., 2017). Así, un estudiante puede requerir 3 tipos de apoyos: conocimientos o habilidades generales, conocimientos específicos de la carrera, o apoyos vocacionales (que ajusten sus expectativas de la carrera con lo que es realmente). Respecto de las habilidades generales, es ampliamente reconocido que el acceso a la Universidad supone el ingreso a una nueva cultura (Muñoz et al., 2016) y que en ésta, las habilidades para leer y escribir propias de la enseñanza secundaria pueden no ser suficientes para abordar con éxito las exigencias de la formación universitaria. En efecto, leer y escribir en la Universidad supone aprovechar el potencial epistémico de ambas prácticas para no solo acceder al conocimiento, sino también para transformarlo.

De igual manera, se evidencia la necesidad de apoyos en conocimientos específicos, normalmente asociados a asignaturas "críticas" que requieren de conocimientos previos más avanzados para poder transitar con éxito en su formación disciplinar, por ejemplo en el área de matemática (Cf. Cifuentes et al., 2017). Finalmente, los estudiantes pueden demandar también apoyos a nivel de orientación vocacional (Bulgarelli-Bolaños et al., 2017). Diversos estudios valoran el rol de los apoyos vocacionales como una herramienta clave para el desarrollo de transiciones exitosas en la vida universitaria, así como para el fortalecimiento de la persistencia y la disminución del abandono de la vida universitaria.

\section{Apoyos socioemocionales}

Un tercer tipo de apoyos asociados al desempeño académico tiene que ver con aspectos socioemocionales. Este ámbito ha sido identificado como un ámbito particular de riesgo en estudiantes universitarios. Resultados de diversos trabajos dan cuenta de que los estudiantes de educación terciaria demandan apoyos en salud mental; asociados específicamente a cuadros depresivos, ansiedad o estrés (Barrera et al., 2019). En Chile, hay reportes recientes que indican altos niveles en sintomatología depresiva (43.2\%), en ansiedad (46.9\%) y en estés (53\%) y donde estas tres dimensiones están presentes a la vez en un $28 \%$ de la muestra, poniendo de manifiesto además que las mujeres evidenciaron más efectos ansiosos y de estrés, que los varones, no así en la sintomatología depresiva (Barrera et al., 2019). Estos resultados son consistentes con reportes previos en este mismo sentido (MINSAL, 2013).

En la etapa del desarrollo que viven los estudiantes universitarios, el ambiente académico puede ser un estresor importante, debido a las altas exigencias intelectuales, sociales y emocionales que éste trae consigo (Halgravez et al., 2017). En este contexto, el ingreso a la universidad es un escenario propicio para que los jóvenes, especialmente los que presentan factores de riesgo psicosociales previos, puedan manifestar diversos trastornos psicológicos (Barrera et al., 2019). Cabe consignar, sin embargo, que los estudiantes con bajos niveles de bienestar mental no están particularmente inclinados a buscar ayuda formal (institucional) sino más bien a la búsqueda informal, incluso en los casos en que la ayuda profesional es bien valorada (Goodwin et al., 2016).

\section{Diversidad de apoyos requeridos}

La acumulación de estas necesidades configura un escenario que eventualmente podría ser una expresión de potenciales dificultades académicas. En esta perspectiva, a mayor tipo de apoyos requeridos, más frágil sería la condición académica del estudiante. Esta preocupación es especialmente seria en el pregrado, aunque se extiende también a los estudios de postgrado. Así, la capacidad de caracterizar oportunamente las necesidades requeridas, aun cuando éstas sean variadas, puede ser una oportunidad para repensar el diseño y las políticas de apoyo por parte de las instituciones universitarias, especialmente cuando las necesidades planteadas por los estudiantes son amplias y diversas.

\section{METODOLOGÍA}

El presente estudio se basa en un diseño cuantitativo en el cual participaron 734 estudiantes universitarios de distintas áreas de conocimiento (ver Tabla 1) pertenecientes a cuatro instituciones universitarias chilenas, públicas y privadas, con cuatro y cinco años de acreditación, y que corresponden a instituciones de acreditación media. Esto es, un tipo de universidad con rango intermedio de acreditación institucional y que atiende al $57,7 \%$ del estudiantado universitario chileno. La muestra estuvo conformada por estudiantes de ambos sexos (71,1\% mujeres), mayores de 18 años, de las distintas cohortes de ingreso, que contestaron el instrumento de manera voluntaria y cuya edad promedio fue de 22.78 años $(S D=4.81)$. 
Tabla 1. Distribución de la muestra por área de conocimiento

\begin{tabular}{|l|c|c|}
\hline Área de conocimiento & Frecuencia & Porcentaje \\
\hline Administración y Comercio & 61 & 8.3 \\
\hline Agropecuaria & 43 & 5.9 \\
\hline Arte y Arquitectura & 10 & 1.4 \\
\hline Ciencias Básicas & 22 & 3.0 \\
\hline Ciencias Sociales & 114 & 15.5 \\
\hline Derecho & 43 & 5.9 \\
\hline Educación & 213 & 29.0 \\
\hline Humanidades & 11 & 1.5 \\
\hline Salud & 147 & 20.0 \\
\hline Tecnología & 70 & 9.5 \\
\hline Total & 734 & 100 \\
\hline
\end{tabular}

\section{Instrumentos}

El instrumento fue elaborado por el equipo de investigadores, a partir de una indagación previa del proyecto Fondecyt 1181159 que permitió seleccionar las variables consideradas en el estudio. Para indagar en las necesidades percibidas de apoyos académicos se evaluaron siete tipos de apoyos que los estudiantes reconocen como necesarios para su éxito académico. Esto se realizó a través de la pregunta "Los apoyos que requiere un estudiante como yo son...", a la que el o la estudiante respondía, en una escala tipo Likert de 6 puntos, su acuerdo o desacuerdo con la necesidad de apoyo en siete tipos de apoyos específicos: a) Hábitos y técnicas de estudios, b) Gestión del tiempo y del estrés, c) Conocimientos generales, d) Conocimientos específicos de la carrera, e) Necesidades de apoyo respecto a orientación vocacional, f) Apoyo afectivo, y finalmente g) Ayuda en Salud Mental. Estos tipos de apoyo se organizan en torno a los tres tipos de apoyo anteriormente caracterizados: estratégicos (a y b), disciplinares, asociados a la disciplina o profesión (c, d, y e) y socioemocionales ( $\mathrm{y} \mathrm{g}$ ), con buen índice de ajuste (RMSEA= .05, IC [.02-.07]; CFI= .98; TLI= .97).

Por otra parte, el indicador "diversidad de apoyos requeridos" fue operacionalizado como el porcentaje de distintos tipos de apoyos complementarios que los estudiantes perciben como altamente necesarios en sus estudios. Para calcular este índice se recodificaron los ítems de apoyos requeridos, generando variables dummy (0-1) en donde aquellos estudiantes que en una escala de 1 a 6 marcaron las opciones de 5 y 6 (tercio superior de la escala), fueron identificados como aquellos estudiantes que claramente manifiestan requerir de ese tipo de apoyo. Evidentemente, esta es una recodificación conservadora, ya que excluye a aquellos estudiantes que marcaron la alternativa 4 situada por sobre el punto medio de la escala. Con esta medida, aseguramos una aproximación más nítida respecto de la percepción de necesidad de apoyo. Con estas variables dicotómicas se calculó la proporción de apoyos que requieren estos estudiantes respecto de estos siete tipos de apoyos consultados.

\section{Procedimientos}

Los datos fueron recogidos a través de un cuestionario on-line previamente validado por un grupo de expertos. El acceso a la muestra fue intermediado por las distintas instituciones, quienes enviaron una invitación a los estudiantes de pregrado a través de correo electrónico y cuyo formulario podía ser respondido a través de computador o de teléfono portátil, para evitar el eventual sesgo que implicaba el acceso a un computador. Tras leer y dar su acuerdo al consentimiento informado, los estudiantes accedían al cuestionario, no permitiendo continuar a aquellos estudiantes que habían declarado tener menos de 18 años (minoría de edad), por estar impedidos de consentir legalmente.

\section{RESULTADOS}

A continuación se presentan los resultados respecto a los tipos de apoyos más demandados, la relación entre distintos tipos de apoyos institucionales demandados por los estudiantes, la diversidad de apoyos requeridos, diferencias por sexo, y finalmente, la estabilidad de los requerimientos de apoyo a través del tiempo.

\section{Tipos de apoyos más demandados}

Al comparar los tres tipos de apoyos requeridos constatamos que hay diferencias significativas entre cada uno de ellos $(p<.001)$. El tipo más demandado son los apoyos estratégicos $(\mathrm{AE})$ con una media de $5.07(\mathrm{SD}=$ 1.13) seguido de los apoyos socioafectivos (ASA) cuya media alcanza $4.63(S D=1.47)$ y finalmente de los apoyos disciplinares (AD) con una media de $4.37(S D=1.30)$. No obstante, y a fin de aportar con una información más detallada, analizamos cada uno de los apoyos individuales evaluados que son percibidos como necesarios para los estudiantes. 
Tabla 2: Jerarquía de necesidad de apoyos por tipo. Medias y subconjuntos homogéneos $(p>.05)$

\begin{tabular}{|l|c|c|c|c|c|c|}
\hline \multicolumn{1}{|c|}{ Variables / Subconjuntos } & 1 & 2 & 3 & 4 & 5 & 6 \\
\hline Gestión del tiempo y del estrés (AE) & 5.19 & & & & & \\
\hline Hábitos y técnicas de estudios (AE) & & 5.02 & & & & \\
\hline Ayuda en Salud Mental (ASE) & & & 4.76 & & & \\
\hline Conocimientos específicos de mi carrera (AD) & & & 4.68 & 4.68 & & \\
\hline Conocimientos generales(AD) & & & & 4.58 & 4.58 & \\
\hline Apoyo afectivo (ASA) & & & & & 4.50 & \\
\hline Orientación vocacional(AD) & & & & & & 3.75 \\
\hline
\end{tabular}

A partir de una prueba de comparación de medias, se establecieron seis subgrupos homogéneos o niveles de necesidad (ver Tabla 2). Así, la Gestión del tiempo y del estrés, y la necesidad de apoyos en hábitos y técnicas de estudios, aparecen como los dos principales tipos de apoyo requeridos. En un tercer nivel, sin diferencias significativas entre ellos se sitúan la demanda de ayuda en salud mental y el apoyo respecto a conocimientos específicos de la carrera. Tras ellos, aparece la necesidad de conocimientos generales y apoyo afectivo. Finalmente, con una media muy inferior, se sitúa la necesidad percibida de apoyo en orientación vocacional distanciada claramente del promedio de los distintos tipos de apoyos requeridos (ver Figura 1).
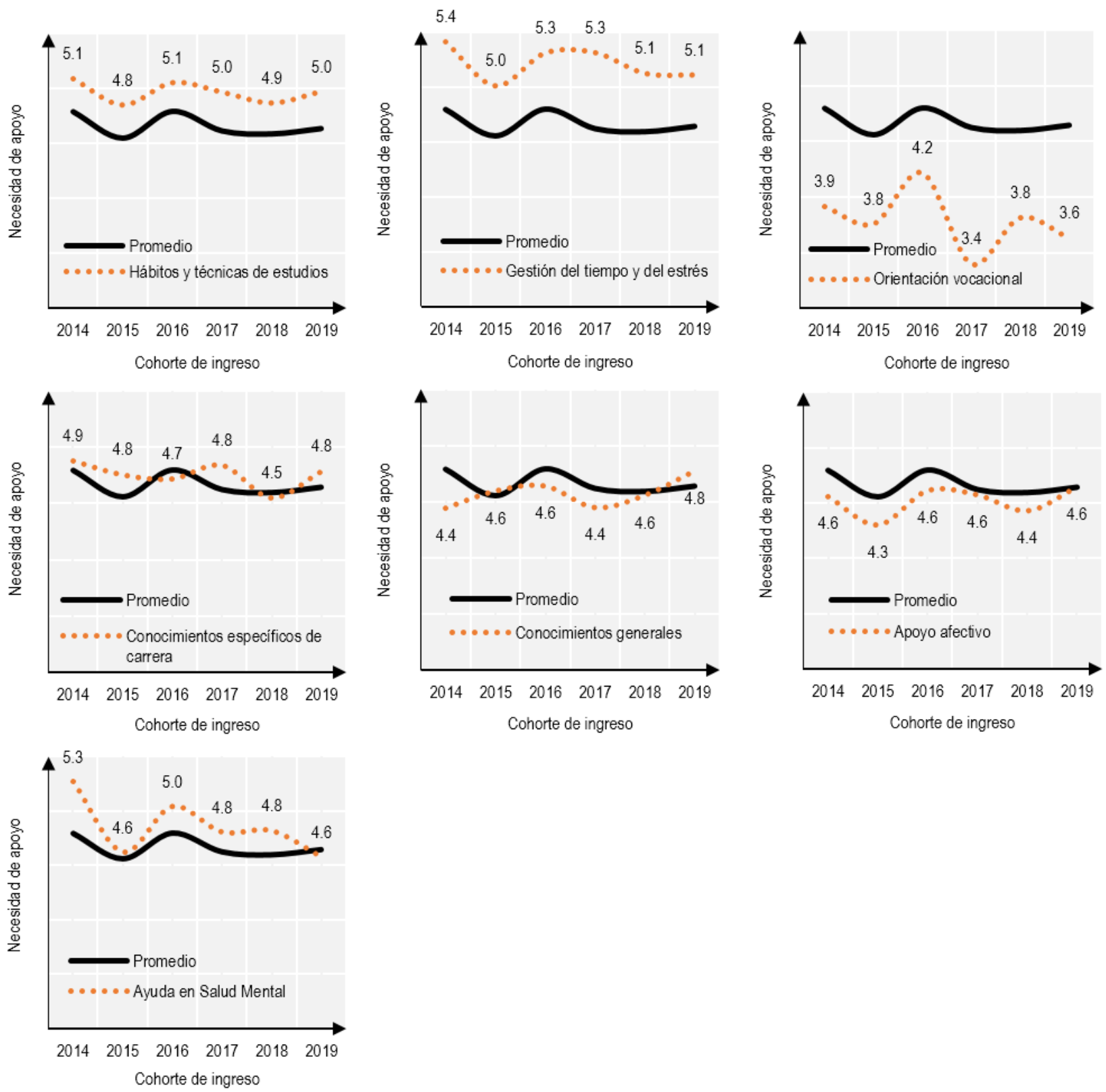

Fig. 1: Necesidad de apoyo por tipo y cohorte de ingreso 


\section{Relación entre Distintos Tipos de Apoyos}

Si bien existe una relación significativa entre todos los tipos de apoyos demandados por los estudiantes (ver Tabla 3) la relación es mucho más fuerte entre la demanda de apoyos respecto a hábitos de estudios y gestión del tiempo y del estrés $(r=.532)$; entre la necesidad de apoyos en conocimientos específicos de la carrera y en orientación vocacional $(r=.492)$ y respecto a apoyos en conocimientos generales $(r=.568)$; y entre la necesidad de apoyo en salud mental y apoyo afectivo $(r=.596)$.

Tabla 3: Matriz de correlaciones entre distintos tipos de apoyos requeridos

\begin{tabular}{|c|c|c|c|c|c|c|}
\hline & 1 & 2 & 3 & 4 & 5 & 6 \\
\hline 1. Hábitos y técnicas de estudios & - & & & & & \\
\hline 2. Gestión del tiempo y del estrés & $.532^{\star * \star}$ & - & & & & \\
\hline 3. Conocimientos generales & $.286^{\star * \star}$ & $.229^{\star \star \star}$ & - & & & \\
\hline 4. Conocimientos específicos & $.262^{* \star *}$ & $.291^{\star \star \star}$ & $.568^{\star * \star}$ & - & & \\
\hline 5. Orientación vocacional & $.222^{\star * \star}$ & $.274^{\star \star \star}$ & $.452^{\star \star \star}$ & $.492^{\star \star \star}$ & - & \\
\hline 6. Apoyo afectivo & $.280^{\star * \star}$ & $.351^{\star \star \star}$ & $.324^{\star * \star}$ & $.252^{* \star *}$ & $.319^{\star \star \star}$ & - \\
\hline 7. Ayuda en Salud Mental & $.220^{* * *}$ & $.406^{\star * \star}$ & $.215^{\star * *}$ & $273^{* \star *}$ & $.248^{\star \star *}$ & $.596^{\star \star \star}$ \\
\hline
\end{tabular}

Con todo, nos interesó especialmente explorar cuáles de ellos explicaban la necesidad de apoyos relacionados con salud mental, considerando la covariación que pudiese existir entre las distintas variables. Para ello se realizó una regresión múltiple (step-wise) utilizando esta variable como dependiente y los otros seis tipos de apoyos demandados como factores. Los resultados muestran que el modelo más parsimonioso no tiene que ver con carencias académicas (hábitos o conocimientos), sino con la necesidad de apoyo afectivo $\left(r^{2}\right.$ adj. $\left.=.366, p<.001\right)$. Los restantes modelos significativos incrementan en un $5.1 \%$ la varianza explicada alcanzando un $r^{2}$ adj. $=.417, p<.001$, fruto de la incorporación de las variables Gestión del tiempo y del estrés $(p<.001)$, Conocimientos específicos de mi carrera $(p<.001)$, Conocimientos generales $(p=.026)$, y Hábitos y técnicas de estudios $(p=.044)$.

Tabla 4: Resumen del modelo de regresión (Ayuda en salud mental)

\begin{tabular}{|c|l|c|c|c|c|}
\hline Modelo & & $R$ & $R^{2}$ & $\begin{array}{c}R^{2} \\
\text { corregida }\end{array}$ & $\begin{array}{c}\text { Error típico de la } \\
\text { estimación }\end{array}$ \\
\hline 1 & Apoyo afectivo & $.606^{\mathrm{a}}$ & .367 & .366 & 1.28 \\
\hline 2 & Apoyo afectivo + Gestión del tiempo y del estrés & $.637^{\mathrm{b}}$ & .406 & .404 & 1.24 \\
\hline 3 & $\begin{array}{l}\text { Apoyo afectivo + Gestión del tiempo y del estrés + } \\
\text { Conocimientos específicos }\end{array}$ & $.642^{\mathrm{c}}$ & .412 & .409 & 1.24 \\
\hline 4 & $\begin{array}{l}\text { Apoyo afectivo + Gestión del tiempo y del estrés + } \\
\text { Conocimientos específicos + Conocimientos generales }\end{array}$ & $.647^{\mathrm{d}}$ & .418 & .414 & 1.23 \\
\hline 5 & $\begin{array}{l}\text { Apoyo afectivo + Gestión del tiempo y del estrés + } \\
\text { Conocimientos específicos + Conocimientos generales + } \\
\text { Hábitos y técnicas de estudios }\end{array}$ & $.650^{\mathrm{e}}$ & .422 & .417 & 1,23 \\
\hline
\end{tabular}

\section{Diversidad de Apoyos Requeridos}

Tras la recodificación en variables dummy de los diferentes tipos de apoyos que los estudiantes declaran necesitar se construyó un índice de diversidad de apoyos requeridos, que da cuenta de la amplitud de la demanda expresado como el porcentaje de apoyos distintos son percibidos con alta demanda (puntuaciones 5 y 6). Los resultados muestran que mientras un $4.4 \%$ de los estudiantes declara no requerir ningún apoyo adicional, un $31 \%$ requieren entre uno y tres tipos de apoyos, mientras que un $68,6 \%$ de los estudiantes requiere apoyo en cuatro o más de las dimensiones evaluadas. En la misma línea, esta vez segmentado por sexo, se observa que las mujeres perciben mayor necesidad de apoyos que los varones $F_{(1,765)}=10.975, p=$ .001 , con un promedio de 4.57 dimensiones requeridas de un total de 7 respecto a sus pares varones quienes requieren 4.02 .

Tabla 5: Índice de apoyos requeridos. (Apoyos requeridos sobre un total de 7 tipos diferentes)

\begin{tabular}{|c|c|c|c|c|}
\hline \multicolumn{2}{|c|}{$\%$ apoyos requeridos } & Frecuencia & $\%$ & \% acumulado \\
\hline 0 & $(0$ de 7$)$ & 32 & 4.4 & 4.4 \\
\hline 14.29 & $(1$ de 7$)$ & 46 & 6.3 & 10.6 \\
\hline 28.57 & $(2$ de 7$)$ & 58 & 7.9 & 18.5 \\
\hline 42.86 & $(3$ de 7$)$ & 94 & 12.8 & 31.3 \\
\hline 57.14 & $(4$ de 7$)$ & 127 & 17.3 & 48.6 \\
\hline 71.43 & $(5$ de 7$)$ & 114 & 15.5 & 64.2 \\
\hline 85.71 & $(6$ de 7$)$ & 121 & 16.5 & 80.7 \\
\hline 100.00 & $(7$ de 7$)$ & 142 & 19.3 & 100 \\
\hline Total & & 734 & 100 & 100 \\
\hline
\end{tabular}




\section{Diferencias por Sexo}

El análisis por sexo muestra que las mujeres declaran necesitar significativamente más apoyos que los varones de la muestra respecto a Hábitos y técnicas de estudios $F_{(1,729)}=14,685, p<.001$; Gestión del tiempo y del estrés $F_{(1,727)}=20,933, p<.001$; Apoyo afectivo $F_{(1,729)}=16.236, p<.001$ y Ayuda en Salud Mental $F_{(1,728)}=$ $27,282<.001$. En el resto de los tipos de apoyo, no se observaron diferencias significativas $(p>.01)$. Cabe destacar igualmente que en los casos de las ayudas referidas a hábitos y técnicas de estudio, gestión del tiempo y del estrés, apoyo afectivo y ayuda en salud mental, la dispersión de los datos es mayor en el grupo de mujeres. Esta falta de homocedasticidad de las varianzas (Levene <.05), en las variables con diferencias significativas, sugiere tomar con cautela estos resultados. Algo similar se puede observar cuando se comparan los porcentajes de mujeres y varones con puntuaciones altas ( $>4$, en escala de 1 a 6 ) para las variables Gestión del tiempo y el estrés, Apoyo afectivo y demanda de ayuda en salud mental $(p<.01 ; p<.01$ y $p<.001$, respectivamente).

Tabla 6: Descriptivos de Requerimiento por Tipos de apoyos

\begin{tabular}{|c|c|c|c|c|c|c|}
\hline & \multicolumn{3}{|c|}{ Muestra general } & \multicolumn{3}{|c|}{$\begin{array}{l}\text { Alta demanda en \% } \\
\text { (puntuaciones }>4 \text { ) }\end{array}$} \\
\hline & $M$ & Mujeres & Varones & $M(\%)$ & Mujeres & Varones \\
\hline Hábitos y técnicas de estudios & 5.03 & 5.13 & $4.76^{*}$ & 74.09 & 76.26 & 68.48 \\
\hline Gestión del tiempo y estrés & 5.19 & 5.33 & $4.83^{* *}$ & 79.14 & 82.57 & $69.70^{\star \star}$ \\
\hline Conocimientos generales & 4.58 & 4.62 & 4.48 & 57.52 & 58.12 & 55.76 \\
\hline Conocimientos específicos & 4.68 & 4.72 & 4.57 & 62.91 & 64.53 & 58.54 \\
\hline Orientación vocacional & 3.75 & 3.74 & 3.76 & 42.03 & 42.17 & 41.82 \\
\hline Apoyo afectivo & 4.50 & 4.66 & $4.05^{\star \star \star}$ & 59.24 & 63.24 & $47.88^{\star \star}$ \\
\hline Ayuda en Salud Mental & 4.76 & 4.95 & $4.25^{\star \star *}$ & 65.84 & 70.32 & $53.33^{\star * *}$ \\
\hline Índice de Diversidad de apoyos & 63.10 & 65.27 & $57.48^{\star *}$ & & & \\
\hline
\end{tabular}

\section{Estabilidad de los Requerimientos de Apoyo}

Finalmente, se evaluó si el requerimiento de apoyo era similar o distinto dependiendo de la etapa de la carrera del estudiante, contrastando las medias para cada cohorte de ingreso. Los resultados obtenidos sobre la evolución de la necesidad de estos apoyos en las cohortes de los últimos 6 años muestran a través del análisis de la varianza, una estabilidad en todos los tipos de apoyos $(p>.05)$ salvo en el caso de la necesidad de apoyos de orden vocacional. En este caso, si bien se observa una caída significativa (menor requerimiento de apoyos en este aspecto) en esta variable entre los años 2016 y 2017 ( $p=.001)$, no hay diferencias significativas entre las restantes cohortes de ingreso (ver Figura 2).

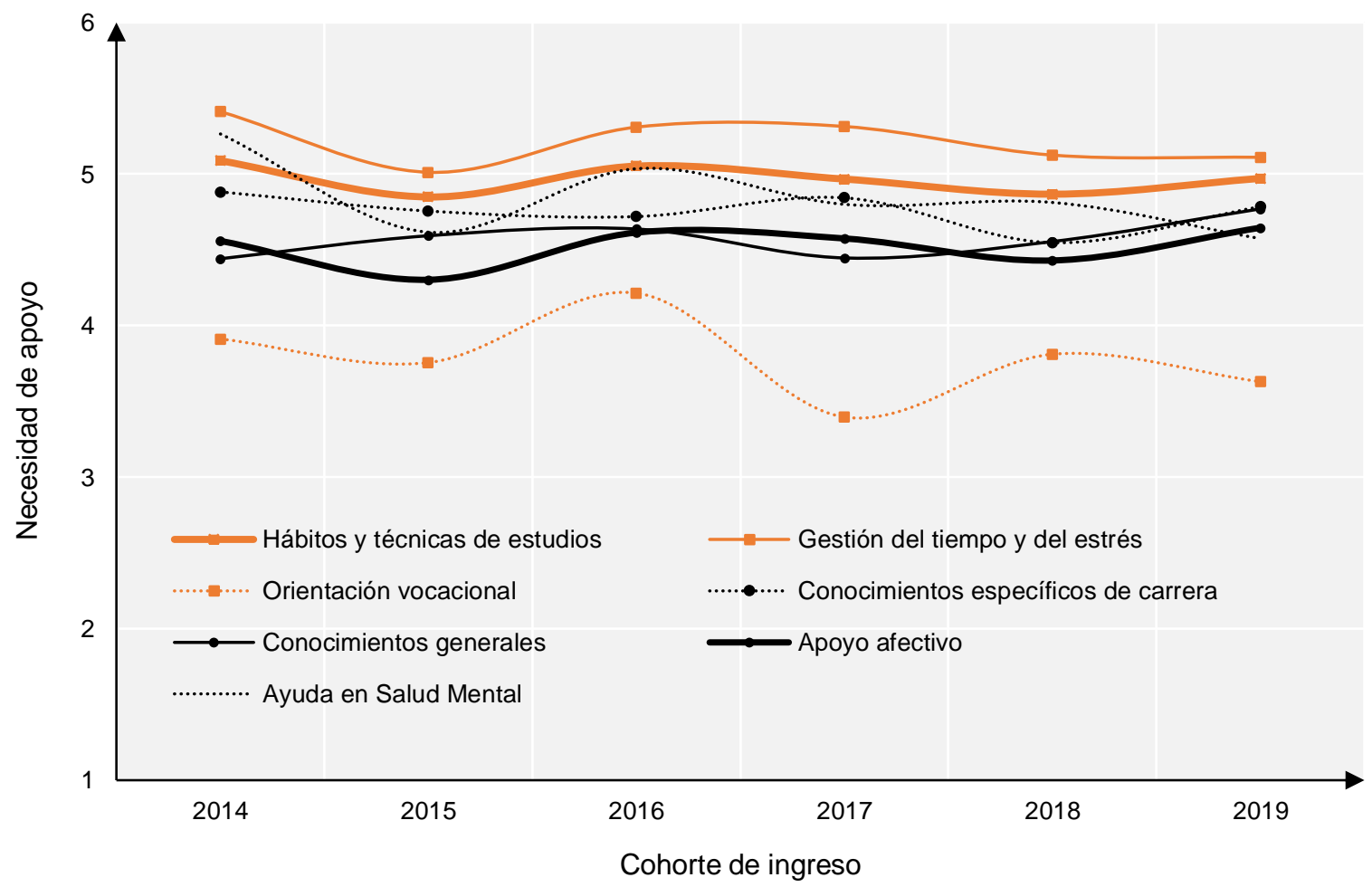

Fig. 2: Tipos de apoyos demandados por cohorte de ingreso 
Al contrastar los requerimientos de apoyo en la submuestra de aquellos que efectivamente han buscado ayuda a nivel institucional y participado algún tipo de dispositivo ofertado por la institución $(n=282)$, el panorama es el mismo. Independientemente de en qué curso se encuentra el estudiante, sus requerimientos de apoyos siguen siendo altos y no se evidencian diferencias por cohorte de ingreso $(p>.075)$. Este panorama se repite si consideramos la cantidad de apoyos altamente requeridos (puntuaciones $>4$ ), en este caso tampoco hay diferencias entre alumnos de las distintas cohortes $(p=.223)$.

\section{DISCUSIÓN}

Este estudio tenía por objetivo caracterizar los apoyos académicos requeridos por estudiantes universitarios para enfrentar con éxito su proceso formativo. Se buscaba así establecer una jerarquía de los tipos de apoyos requeridos y evaluar la amplitud de dichas necesidades. Si bien es posible identificar una jerarquía en los apoyos demandados por los estudiantes, se constatan otras cuatro situaciones que a nuestro juicio, son claves para aquilatar estas demandas: prevalencia en la demanda, la correlación que se observa entre estos siete tipos de apoyos requeridos, la diversidad de apoyos requeridos, y finalmente, cómo esta demanda de apoyos demandados son similares entre las distintas cohortes de ingreso (año que se cursa en la universidad).

Si bien es posible identificar a dos tipos de apoyos como más necesarios, tales como la gestión del tiempo y de estrés, así como los hábitos y técnicas de estudio, y que ambos tipos de apoyos corresponden a búsquedas de apoyos instrumentales con un claro talante adaptativo (Puustinen, 2013), los resultados muestran una alta prevalencia en la demanda en todos los tipos de apoyos evaluados, con la sola excepción de la necesidad del apoyo de orientación vocacional. En este contexto, la intensidad de la demanda de apoyos pareciera no ser suficiente antecedente para dar cuenta de la situación de los universitarios actuales. Los resultados muestran que no son pocas las necesidades de apoyo. Lejos de ello, las necesidades son bastante extendidas. Más del $50 \%$ de los estudiantes de la muestra, requieren cuatro o más tipos distintos de apoyo, mientras que un $35 \%$ de la muestra dice requerir seis o siete tipos distintos de apoyos, sobre un total de siete posibles tipos de apoyos.

Sin embargo, el resultado más preocupante es la estabilidad de estos resultados en las distintas cohortes de ingreso. Es decir, la percepción de necesidad de apoyos no disminuye con los años. Esta estabilidad no solo se observa en los resultados generales sino también en la submuestra de estudiantes que declaran haber participado de alguno de los apoyos ofrecidos por su institución. Esto sugiere que las necesidades de apoyo no están siendo del todo satisfechas. Ello, pese a que la valoración que estos estudiantes tienen de los servicios de apoyo es positiva (sólo un 12,2\% la considera deficiente). Así, pareciera que estos altos y diversos requerimientos de apoyo y la falta de diferencias entre los estudiantes de las distintas cohortes, hablan de un problema que no se soluciona con dispositivos específicos, incluso si estos están bien valorados por los estudiantes.

De esta manera, los tipos de apoyos más prevalentes, la amplitud en esta demanda de apoyos, así como la persistencia del requerimiento a través del tiempo, constituyen un marco para poner en cuestión la estrategia de focalización de los apoyos institucionales, es decir, de que las instituciones identifiquen los apoyos puntuales requeridos por los estudiantes e intenten subsanarlos a través de dispositivos específicos, por ejemplo, dispositivos de nivelación de competencias. Obviamente, no se trata de desconocer el efecto que se observa, por ejemplo, en los apoyos mediados por pares (Vaessen et al., 2014). Los resultados más bien apuntan a replantearse esta estrategia y abrirse a la posibilidad de un apoyo más integral que ponga énfasis en la incorporación de estos estudiantes a la dinámica específica que significan los estudios en la educación superior.

Por otra parte, los resultados muestran que algunos de estos apoyos son requeridos de manera significativamente más elevada por las mujeres. Esto es consistente con los resultados de Barrera et al. (2019), que muestran que las mujeres universitarias presentan significativamente más síntomas de ansiedad y estrés. Asimismo, una mirada más profunda a la percepción de necesidad de apoyos, indica que, en principio, la necesidad de apoyos en salud mental se relaciona primariamente con necesidades de apoyo en el ámbito afectivo y secundariamente con otros tipos de apoyos que pudiesen vincularse más directamente con una sobre exigencia o sobrecarga vinculada a una transición no exitosa a una nueva cultura (manejo del estrés, estrategias y hábitos de estudio, o conocimientos específicos). No obstante, cabe señalar que estudios como los de Olivarí y Mellado (2019) muestran que detrás de la demanda de ayuda, especialmente en salud mental, en realidad se esconden otros requerimientos. A lo anterior hay que agregar, el hecho de que la toma de datos de nuestro estudio coincide con un año (2019) en el cual la temática de la salud mental tuvo una cobertura mediática muy importante (e.g. González, 2019).

La evidencia aportada por este estudio sugiere que los apoyos que las instituciones universitarias brindan a nivel académico debiera re-pensarse de una manera más integral y menos focalizada en apoyos concretos 
(cf. McCabe, 2018), por ejemplo, a partir de lógicas introductorias a la vida universitaria que permitan a los estudiantes el tiempo para realizar esta transición y fortalecer el desarrollo de funciones ejecutivas que favorezcan procesos de mayor complejidad en el aprendizaje. El alto nivel de demanda en todos los tipos de apoyos consultados, la diversidad de apoyos que requieren nuestros estudiantes y lo estable que continúa siendo esta demanda durante la formación universitaria, constituyen una voz de alerta para asumir el desafío formativo de una manera más integral. Los apoyos puntuales parecen no dar respuesta cabal a un problema, que a todas luces, es mucho mayor que lo que puede solucionar una iniciativa puntual y focalizada como las que las instituciones universitarias chilenas despliegan. Ello, evidentemente, implica más tiempo, pero apunta a lograr resultados de inclusión y calidad a los que todos aspiramos.

Como todo estudio, la presente indagación no está exenta de limitaciones. Por su carácter exploratorio, se prioriza la diversidad de la muestra por sobre un diseño cuasi experimental con grupos con tamaños similares. A pesar que en la mayoría de los casos hay homogeneidad de la varianza, en próximos estudios comparativos sería importante incorporar diseños que permitan una comparación más fina entre subgrupos.

\section{CONCLUSIONES}

De acuerdo al trabajo presentado y a los resultados obtenidos, se pueden plantear las siguientes conclusiones principales:

1. Los apoyos de carácter estratégico instrumental son el principal requerimiento de apoyo en el marco académico, por parte de los estudiantes.

2. La demanda de apoyo académico en este tipo de estudiante es amplia (muchos tipos de apoyos requeridos) y elevada (alta demanda de cada uno de estos tipos de apoyo)

3. El requerimiento de apoyo no varía durante la formación universitaria. Ello apoya la idea de repensar las estrategias de apoyo académico en la formación universitaria.

\section{MATERIAL SUPLEMENTARIO}

Los datos que apoyan los resultados de este estudio están disponibles y pueden ser solicitados al autor de correspondencia.

\section{AGRADECIMIENTOS}

Los autores agradecen el financiamiento de este artículo por parte de la Agencia Nacional de Investigación y Desarrollo, ANID - Chile, a través del Proyecto Fondecyt Regular 1181159. Agradecemos igualmente a Marcelo González Figueroa por el apoyo en la representación gráfica de los datos.

\section{REFERENCIAS}

Barrera, A., Neira, M., y otros tres autores, Apoyo social percibido y factores sociodemográficos en relación con los síntomas de ansiedad, depresión y estrés en universitarios chilenos, https://doi.org/10.5944/rppc.23676, Revista de Psicopatología y Psicología Clínica, 24(2), 105-115 (2019)

Barrera, A., y Vinet, E.V., Adultez emergente y características culturales de la etapa en universitarios chilenos, http://dx.doi.org/10.4067/S0718-48082017000100005, Terapia Psicológica, 35(1), 47-56 (2017)

Bickerdike, A., O'Deasmhunaigh, C., O'Flynn, S., y O'Tuathaigh, C., Learning strategies, study habits and social networking activity of undergraduate medical students, https://doi.org/10.5116/ijme.576f.d074, International Journal of Medical Education, 7, 230-236 (2016)

Black, S., y Allen, J.D., Academic help seeking, https://doi.org/10.1080/02763877.2018.1533910, The Reference Librarian, 60, 62-76 (2019)

Bulgarelli-Bolaños, R.M., Rivera-Rodríguez, J.A., y Fallas-Vargas, M.A., El proceso vocacional del estudiantado universitario en condición de logro y rezago académico: un análisis desde el enfoque evolutivo de Donald Super, http://dx.doi.org/10.15359/ree.21-1.1, Revista Electrónica Educare, 21, 1-24 (2017)

Casanova, J.R., Cervero, A., y otros tres autores, Factors that determine the persistence and dropout of university students, https://doi.org/10.7334/psicothema2018.155, Psicothema, 30(4), 408-414 (2018)

Chang, J., Wang, S-W., y otros tres autores, The complexity of cultural mismatch in higher education: norms affecting first-generation college students' coping and help-seeking behaviors, https://doi.org/10.1037/cdp0000311, Cultural Diversity and Ethnic Minority Psychology, 26(3), 280-294 (2020)

Chowdhury, S., y Halder, S., Academic help-seeking: a constructivist approach in learning and achievement, International Journal of Education and Management Studies, 9(4), 227-231 (2019) 
Chrysikos, A., Ahmed, E., y Ward, R., Analysis of Tinto's student integration theory in first-year undergraduate computing students of a UK higher education institution, https://doi.org/10.1108/IJCED-10-2016-0019, International Journal of Comparative Education and Development, 19(2/3), 97-121 (2017)

Cifuentes, M.B., Munizaga, F.R., y Mella Luna, J., Más tiempo para aprender: evidencias para aportar al debate sobre equidad, inclusión y gratuidad de la educación superior a partir de resultados de dispositivos de nivelación matemática, https://doi.org/10.7764/PEL.54.1.2017.1, Pensamiento Educativo, 54(1), 1-15 (2017)

Coertjens, L., Brahm, T., Trautwein, C., y Lindblom-Ylänne, S., Students' transition into higher education from an international perspective, https://doi.org/10.1007/s10734-016-0092-y, Higher Education, 73(3), 357-369 (2017)

Collins, W., y Sims, B.C., Help seeking in higher education academic support services, in Help seeking in academic settings: goals, groups, and contexts by S. Karabenick, y R.S. Newman (Eds.), pp. 203-223, Routledge, Londres, Inglaterra (2006)

Engstrom, C., y Tinto, V., Access without support is not opportunity, https://doi.org/10.3200/CHNG.40.1.46-50, Change, 40(1), 46-50 (2008)

Etxeberria, P., Alberdi, E., Eguia, I., y García, M.J. Análisis del rendimiento académico en relación al perfil de ingreso del alumnado e identificación de carencias formativas en materias básicas de dos grados de ingeniería, https://dx.doi.org/10.4067/S0718-50062017000400007, Formación Universitaria, 10, 67-74 (2017)

González, V., Protesta de estudiantes de Arquitectura de la FAU por sobrecarga reabre debate por salud mental. Biobiochile.cl, https://n9.cl/38qf, (2019)

Goodwin, J., Behan, L., y otros tres autores, Help-seeking behaviors and mental well-being of first year undergraduate university students, https://doi.org/10.1016/j.psychres.2016.09.015, Psychiatry Research, 246, 129-135 (2016)

Halgravez, L.A., Salinas, J.E., Martínez, G.I., y Rodríguez, O.E., Percepción de estrés en universitarios, su impacto en el desempeño académico, afrontamiento y apoyo familiar, Revista Mexicana de Estomatología, 3(2), 27-36 (2017)

Hussey, T., y Smith, P., Transitions in higher education, https://doi.org/10.1080/14703291003718893, Innovations in Education and Teaching International, 47(2), 155-164 (2010)

Jeffery, D., y Johnson, D., Whose fault is failure? Contested perspectives of academic support in tertiary educational institutions in South Africa, https://doi.org/10.1177/1745499919864731, Research in Comparative and International Education, 14(3), 376-393 (2019)

Larose, S., Cyrenne, D., y otros cuatro autores, Personal and social support factors involved in students' decision to participate in formal academic mentoring, https://doi.org/10.1016/j.jvb.2008.11.002, Journal of Vocational Behavior, 74(1), 108-116 (2009)

McCabe, J.A., What learning strategies do academic support centers recommend to undergraduates?, https://doi.org/10.1016/j.jarmac.2017.10.002, Journal of Applied Research in Memory and Cognition, 7(1), 143-153 (2018)

Micin, S., Carreño, B., y Urzúa, S., Perfil de ingreso de estudiantes en carreras del área de la salud, Educación Médica Superior, 31(3), 155-167 (2017)

MINSAL., Guía clínica depresión en personas de 15 años y más, https://www.minsal.cl, Ministerio de Salud, Santiago, Chile (2013)

Morandi, G., Ungaro, A.M., Arce, D., y Gallo, L., Los procesos de afiliación académica en el ingreso a la universidad pública: la experiencia estudiantil, Actas de Periodismo y Comunicación, 5(2), 1-16 (2019)

Muñoz, C., Valenzuela, J., Avendaño, C., y Núñez, C., Mejora en la motivación por la lectura académica: la mirada de estudiantes motivados, http://doi.org/10.18239/ocnos_2016.15.1.941, Ocnos, 15(1), 52-68 (2016)

Olivari, C., y Mellado, C., Reconocimiento de trastornos de salud mental en adolescentes escolarizados: estudio descriptivo, https://doi.org/10.5867/medwave.2019.03.7617, Medwave, 19(3), 7617 (2019)

Parnes, M.F., Kanchewa, S.S., Marks, A.K., y Schwartz, S.E., Closing the college achievement gap: impacts and processes of a help-seeking intervention, https://doi.org/10.1016/j.appdev.2020.101121, Journal of Applied Developmental Psychology, 67, 101121 (2020)

Peeters, A., Robinson, V., y Rubie-Davies, C., Theories in use that explain adolescent help seeking and avoidance in mathematics, https://doi.org/10.1037/edu0000423, Journal of Educational Psychology, 112(3), 533-550 (2020)

Puustinen, M., La demande d'aide chez l'élève: avancées conceptuelles, méthodologiques et nouvelles données, Éditions L'Harmattan, Paris, Francia (2013)

Rascovan, S., La orientación vocacional como experiencia subjetivante, Paidós, Buenos Aires, Argentina, (2016)

Schneider, M., y Preckel, F., Variables associated with achievement in higher education: a systematic review of metaanalyses, https://doi.org/10.1037/bul0000098, Psychological Bulletin, 143(6), 565-600 (2017)

Seamark, D., y Gabriel, L., Barriers to support: a qualitative exploration into the help-seeking and avoidance factors of young adults, https://doi.org/10.1080/03069885.2016.1213372, British Journal of Guidance \& Counselling, 46(1), 120$131(2018)$ 
Seyi-Oderinde, D.R., Influence of socio-demographic factors on the beliefs and attitudes of male students towards helpseeking in universities, Journal of Education Research and Rural Community Development, 2(2), 1-14 (2020)

Silva, M., The pedagogical dimension of equity in higher education, https://doi.org/10.14507/epaa.28.5039, Education Policy Analysis Archives, 28(46), 1-27 (2020)

Vaessen, B.E., Prins, F.J., y Jeuring, J., University students' achievement goals and help-seeking strategies in an intelligent tutoring system, https://doi.org/10.1016/j.compedu.2013.11.001, Computers \& Education, 72, 196-208 (2014) 